\title{
The Effect of Bariatric Surgery and Endoscopic Procedures on Gastroesophageal Reflux Disease
}

\author{
Ofer Z Fass $^{1 *}$ and Hiroshi Mashimo ${ }^{2}$ \\ ${ }^{I}$ Department of Medicine, New York University Langone Health, New York, NY, USA and ${ }^{2}$ Department of Medicine, VA Boston Healthcare \\ System, Harvard Medical School, Boston, MA, USA
}

Obesity is a global epidemic posing a significant burden on patients and healthcare systems. Gastroesophageal reflux disease is associated with obesity and its prevalence is also growing worldwide. Numerous bariatric surgeries and endoscopic procedures have arisen to assist with weight loss and management of obesity-related conditions. However, the effect of these interventions on reflux is variable and the evidence is often conflicting. To date, Roux-en-Y gastric bypass remains the gold-standard for attaining both reflux and weight loss management, however novel endoscopic techniques are quickly becoming more prevalent as an alternative to surgery. This review aims to summarize currently available endoscopic and surgical weight loss procedures and their impact on reflux symptoms while emphasizing areas requiring additional investigation.

(J Neurogastroenterol Motil 2021;27:35-45)

Key Words

Bariatrics; Gastrectomy; Gastroesophageal reflux; Obesity

\section{Introduction}

Obesity is a complex medical condition posing a significant burden on our healthcare system. An estimated $\$ 190$ billion is spent annually on obesity-related expenditures, comprising approximately 21 percent of United States health care spending. ${ }^{1}$ It is estimated that greater than one-third of American adults are classified as obese, defined as a body mass index (BMI) $\geq 30 \mathrm{~kg} / \mathrm{m}^{2}$. This percentage is anticipated to surpass $44.0 \%$ of the population with obesity-related healthcare costs reaching $\$ 48$ to $\$ 66$ billion by the year 2030.,4

Gastroesophageal reflux disease (GERD), is a common gastrointestinal illness. Like obesity, GERD was also recently reported to affect approximately a third of American adults, and its prevalence is steadily growing. ${ }^{5}$ GERD is defined as the reflux of stomach contents into the esophagus, often resulting in bothersome symptoms and complications. Reported symptoms are diverse and include retrosternal burning (most common), regurgitation, dysphagia, chest pain, hypersalivation, globus sensation, and nausea. ${ }^{6,7} \mathrm{Com}-$ plications of longstanding GERD can result in significant morbidity, including esophageal strictures, erosive esophagitis, Barrett's esophagus, laryngitis, and laryngeal stricture. ${ }^{8}$ Of greatest concern is Barrett's esophagus, which predisposes to the development of esophageal adenocarcinoma. ${ }^{9}$

There is a well-established relationship between GERD and obesity, with heavier patients experiencing a greater frequency of heartburn symptoms and a higher likelihood of GERD-related

Received: July 31, 2020 Revised: October 24, 2020 Accepted: November 10, 2020

(c) This is an Open Access article distributed under the terms of the Creative Commons Attribution Non-Commercial License (http://creativecommons. org/licenses/by-nc/4.0) which permits unrestricted non-commercial use, distribution, and reproduction in any medium, provided the original work is properly cited.

*Correspondence: Ofer Z Fass, MD

Department of Medicine, New York University Langone Health, 550 1st Ave, NBV 16N30, New York, NY 10016, USA

Tel: +1-520-664-5023, Fax: +1-929-455-9235, E-mail: oferfass@gmail.com 
complications. Questionnaire data from 10545 female participants of the Nurses' Health Study demonstrated a dose-dependent correlation between an incremental increase in BMI and worsening severity and frequency of symptoms $(P<0.001) .{ }^{10}$ Even among participants with a normal BMI, an increase in weight was associated with greater reflux frequency. Further supporting the association between obesity and GERD is a prospective cohort study of 332 patients enrolled in a structured weight loss program at a single academic medical center. ${ }^{11}$ Among the participants, $37.0 \%$ had baseline GERD symptoms with a mean symptom score of 5.5. After 6 months, participants lost an average of $13 \mathrm{~kg}$ and the prevalence of GERD decreased to $15.0 \%(P<0.01)$. Furthermore, $65.0 \%$ of previously affected patients reported complete resolution of their symptoms.

The increasing rate of obesity is projected to cause a concurrent rise in the prevalence of GERD. Thus, through its association as an independent risk factor for GERD, obesity increases the risk of developing Barrett's esophagus and subsequently esophageal adenocarcinoma. ${ }^{12}$ To better understand the relationship between obesity and GERD, it is necessary to examine how obesity augments the pathophysiology of GERD.

\section{Obesity and Gastroesophageal Reflux Disease}

Various mechanisms have been proposed to describe the effect of obesity in augmenting GERD pathophysiology. First is the increased prevalence of hiatal hernias within the obese population. ${ }^{13}$ Hiatal hernias cause the lower esophageal sphincter (LES) to be displaced superiorly into the thoracic cavity. This predisposes the LES to negative intrathoracic pressure, drawing gastric acid into the esophageal lumen and forming a gastric acid pocket above the diaphragm and up against the LES. This acid pocket is difficult for the stomach to clear. Furthermore, hiatal hernias disrupt the crural ligament, resulting in loss of added reinforcement to the LES. ${ }^{14}$ Studies have shown a direct correlation between hiatal hernia size and severity of reflux symptoms. ${ }^{15}$ Obesity is thought to increase the risk of hiatal hernia formation by increasing the intra-abdominal pressure, forcing the gastric fundus up through structural weaknesses in the diaphragm. ${ }^{16}$ The prevalence of hiatal hernias among morbidly obese patients is predicted to be nearly $40 \%$. $^{17}$

Increased intra-abdominal pressure alone, in the absence of hiatal hernia, is also thought to contribute to the development of reflux. A study by Mercer et $\mathrm{al}^{18}$ demonstrated an increased gastroesophageal pressure gradient across the LES and increased esophageal transit times in obese patients when compared to normal-weight patients. Pandolfino et $\mathrm{al}^{19}$ confirmed Mercer's findings by employing a high-resolution esophageal manometry probe and showing a positive correlation between both BMI and waist circumference with intra-abdominal pressure. A recent study of obese individuals (BMI $>35 \mathrm{~kg} / \mathrm{m}^{2}$ ) corroborated findings by both Mercer and Pandolfino by evaluating the relationship between the transdiaphragmatic pressure gradient and GERD. ${ }^{20}$ The presence of GERD and symptom severity correlated with a high transdiaphragmatic pressure gradient due to increasing abdominal pressures. Interestingly, while elevated abdominal pressure was associated with abdominal circumference, there was no correlation with BMI. The relationship between visceral fat and reflux was further supported by Matsuzaki et $\mathrm{al}^{21}$ in which a study of 2608 individuals demonstrated that men had an odds ratio of 1.21 for reflux esophagitis per $50 \mathrm{~cm}^{2}$ of visceral fat area (95\% CI, 1.01-1.46) and women had an odds ratio of 2.31 per $50 \mathrm{~cm}^{2}$ of visceral fat (95\% CI, 1.57-3.40).

Obstructive sleep apnea (OSA) is a common comorbidity in obese patients that is strongly associated with reflux. Surveys have demonstrated that $75 \%$ of patients with OSA suffer from nighttime GERD symptoms compared to $14 \%$ of the United States population. ${ }^{22}$ The mechanism by which OSA is hypothesized to contribute to reflux includes increased intraabdominal pressure and negative intrathoracic pressure. During apneic episodes, inspiratory efforts produce substantial intrathoracic negative pressure, disrupting the LES. Corresponding increases in abdominal pressure results in an elevated transdiaphragmatic gradient, promoting the transit of gastric contents into the esophageal lumen. ${ }^{23}$ The use of continuous positive airway pressure (CPAP) may improve GERD and has been shown to reduce reflux frequency both in patients with and without OSA. ${ }^{24}$ Studies have demonstrated that CPAP use decreases esophageal acid contact time, reduces transient lower esophageal sphincter frequency, and maintains baseline LES pressure. ${ }^{25,26} \mathrm{Ad}-$ ditionally, OSA-related nighttime disturbances cause poor sleep quality, which may contribute to esophageal hypersensitivity and worsening reflux symptoms. ${ }^{27}$

Another proposed mechanism for the development of GERD in obese patients is diet. Consumption of excessive fat promotes the secretion of hormones such as secretin and cholecystokinin, which in turn stimulate LES relaxation. ${ }^{28}$ A study by Fox et al $^{29}$ demonstrated an increased frequency of reflux symptoms and greater esophageal acid exposure in patients with a high-fat diet compared to a low-fat diet. Multiple additional studies have demonstrated a correlation between chocolate, alcohol, and caffeine intake with GERD symptoms secondary to increased relaxation of the LES. ${ }^{30-32}$ 
Finally, several studies have implicated the adipokines leptin and adiponectin, and the hormone ghrelin, in modulating reflux symptoms. A study of 60 adults with endoscopically confirmed GERD, compared to 20 age- and gender-matched controls, suggested a positive correlation between leptin levels and clinical severity of GERD. ${ }^{33}$ Another study confirmed the association between leptin and GERD but found the opposite relationship with ghrelin. Individuals with elevated ghrelin levels had an odds ratio of 0.364 for having GERD. ${ }^{34}$ A detailed study of 21 adults in Japan further suggested a role for adipokines and metabolic syndrome as a link between obesity and GERD. ${ }^{35}$ Elevated BMI and waist circumference were associated with greater symptom severity and video esophagography $\mathrm{X}$-ray severity scores. In turn, high-density lipoprotein cholesterol, serum adiponectin, and active ghrelin, were all negatively associated with symptom severity and X-ray severity scores.

Although various mechanisms have been proposed for the role of obesity in the development of GERD, it is likely that the numerous mechanisms work together to produce an increased pressure gradient across the LES and impair acid clearance from the esophageal lumen. Figure 1 summarizes the major pathophysiologic mechanisms causing reflux in obesity.

\section{Endoluminal Bariatric Procedures and Gastroesophageal Reflux Disease}

As the prevalence of obesity continues to increase in the United States, bariatric procedures have risen to become an essential tool for disease management. Bariatric surgery has remained the leading procedural anti-obesity treatment with 340770 bariatric surgeries performed in 2011 alone. Nearly a third (101 645) of these surgeries were performed in the United States and Canada. ${ }^{36}$ However, surgery poses inherent risks, and the comorbidities seen in obese patients often place them at even greater jeopardy for surgical complications. Therefore, non-surgical bariatric interventions may be considered for the management of obesity in patients who may not be suitable for surgery or who desire less invasive interventions. As endoluminal bariatric procedures become more widely available, their usage will undoubtedly become more prevalent. Therefore, it is necessary to examine the relationship between these procedures and GERD symptoms.

Endoluminal bariatric procedures serve 4 primary purposes; as an early weight loss intervention for individuals that do not meet criteria for surgical treatment, as a bridge to surgery to reduce operative risk, as a primary bariatric or metabolic treatment, and as a revision treatment for failed surgical procedures. ${ }^{37}$ This review focuses on major endoluminal procedures currently in use or under investigation as primary bariatric treatments. These include intragastric balloons, aspiration therapy, endoluminal vertical gastroplasty, endoscopic gastrointestinal bypass devices, and duodenal mucosal resurfacing.

\section{Intragastric Balloons}

Intragastric balloons have been in use since 1982 internationally and remain the most commonly placed endoscopic device outside of the United States. ${ }^{37,38}$ Not until recently has the intragastric balloon become available in the United States, with both Orbera and ReShape Dual Balloon systems approved by the Food and Drug Administration (FDA) in 2015. Intragastric balloons are endoscopically placed within the stomach and filled with $400 \mathrm{~mL}$ to 700

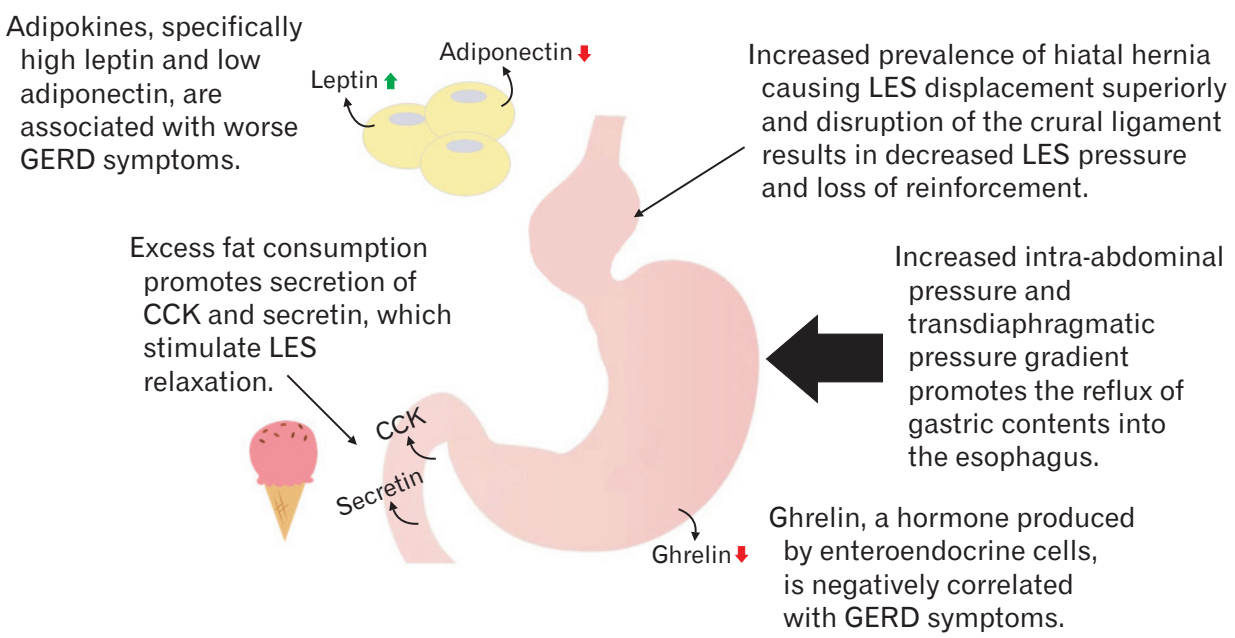

Figure 1. A summary of the major pathophysiologic mechanisms contributing to reflux in obesity. GERD, gastroesophageal reflux disease; CCK, cholecystokinin; LES, lower esophageal sphincter: 
$\mathrm{mL}$ of saline fluid and remain in place for up to 6 months. The mechanism of weight loss is primarily restrictive, limiting the food capacity of the stomach and inducing early satiety. ${ }^{39} \mathrm{~A}$ metanalysis and systematic review identified 5 studies assessing the efficacy of intragastric balloons on weight loss and gastric emptying. Fluidfilled balloons were noted to delay gastric emptying by nearly 2 hours, suggesting an additional mechanism for weight loss. ${ }^{40}$

The relationship between intragastric balloons and GERD symptoms is supported by the literature. The balloon behaves as a space-occupying mass that although intended to induce satiety, also increases the intragastric pressure and the pressure gradient across the LES. Therefore, reflux is a commonly reported adverse effect of the intragastric balloon. A retrospective study of intragastric balloon tolerance and efficacy reported a 6.8\% incidence of de novo GERD following balloon placement. ${ }^{41}$ Many patients respond positively to proton pump inhibitor (PPI) treatment and experts generally recommend placing patients with preexisting GERD on PPI treatment for the entirety of the 6 months the intragastric balloon is in place. $^{41}$

\section{Aspiration Therapy}

In contrast to the intragastric balloon, a novel aspiration device has recently been approved by the FDA which is non-space occupying. The AspireAssist was approved in June 2016 and functions by removing a portion of gastric content following a meal. A gastrostomy-like tube (termed an "A-tube"), is endoscopically placed, allowing for communication between the gastric lumen and skin surface. Approximately 20-30 minutes after eating, the patient drains a portion of their meal through the tube into a receptacle, resulting in the removal of about $30.0 \%$ of the consumed caloric content. $^{42}$

The relationship between GERD symptoms and aspiration therapy is uncertain and requires further investigation. Per FDA summary of safety and effectiveness, 7 patients out of 111 (6.3\%), during randomized control trials reported symptoms of acid reflux or related symptoms of hiccups or belching. ${ }^{43}$ The mechanistic relationship between aspiration therapy and GERD symptoms is unclear; it would be expected that the removal of gastric content via aspiration would decrease gastric acid content and reduce reflux symptoms. As the AspireAssist becomes more widely available, future studies will hopefully further characterize adverse effects and the relationship between aspiration therapy and GERD.

\section{Endoluminal Vertical Gastroplasty}

Endoluminal vertical gastroplasty, also known as endoscopic sleeve gastroplasty, is an endoscopic technique employed to create a narrowed tube of stomach similar to a gastric sleeve. A vacuum is used to suction the anterior and posterior walls of the stomach into proximity and a suturing device is deployed to attach the walls together and form the sleeve. An alternate form of gastroplasty uses a stapler to form the gastric sleeve. ${ }^{44}$ In both cases, the mechanism of weight loss is restrictive, inducing early satiety, and decreased food consumption. A recent meta-analysis of 8 studies evaluating endoluminal vertical gastroplasty demonstrated total body weight loss of $15.1 \%$ at 6 months with a sustained response at 12 months and 18 24 months. ${ }^{45}$

The incidence of reflux following endoluminal vertical gastroplasty is relatively rare. One study evaluated 64 patients at 1-year post-endoluminal vertical gastroplasty and found no incidence of reflux symptoms. Two of the 64 patients had reported reflux symptoms immediately following the procedure, which resolved after 24 hours. ${ }^{46}$ Results from the Transoral Gastric Volume Reduction as Intervention for Weight Management (TRIM) trial show similar results, with 8 patients out of 14 reporting reflux symptoms at baseline prior to endoluminal vertical gastroplasty and the number dropping to 5 out of 14 at 1 -year follow-up. ${ }^{47}$ A more recent study compared endoluminal vertical gastroplasty to laparoscopic sleeve gastrectomy and found that while weight loss at 6 months was less with the endoscopic technique, fewer patients reported new-onset $\operatorname{GERD}(1.9 \%$ vs $14.5 \%, P<0.05){ }^{48}$

\section{Gastrointestinal Bypass Devices}

Endoscopic gastrointestinal bypass devices are investigational and employ the use of a tube that extends either from the proximal duodenum to the mid jejunum, mimicking a duodenojejunal bypass (EndoBarrier; GI Dynamics Inc, Boston, MA, USA), or a tube extending from the gastroesophageal junction to the jejunum (ValenTx; ValenTx Inc, Maple Grove, MN, USA). ${ }^{44}$ Both of these systems induce weight loss by means of bypassing significant portions of the small intestine, thereby causing malabsorption. The largest study evaluating EndoBarrier efficacy was a 73-person multicenter trial in the Netherlands, which demonstrated an average weight loss of $10.6 \mathrm{~kg}$ compared to $5.3 \mathrm{~kg}$ in the control arm. ${ }^{49} \mathrm{In}$ the United States, the ENDO Trial was supposed to evaluate the safety and efficacy of EndoBarrier, however, was stopped early due to the development of liver abscesses in 7 (3.5\%) participants. ${ }^{50}$

\section{Duodenal Mucosal Resurfacing}

An emerging bariatric endoscopic therapy is duodenal mucosal resurfacing (Fractyl Laboratories Inc, Lexington, MA, USA). ${ }^{51}$ 
The device works by employing circumferential hydrothermal ablation to disrupt the presentation of nutrients to the duodenum, thus altering hormonal signaling, ultimately resulting in improved glycemic control and weight loss. A recent open-label study demonstrated that duodenal mucosal resurfacing is generally well-tolerated with modest weight loss and clinically significant improvement in glycemic control at 1 year. ${ }^{52}$ Given the rapid healing of the mucosa after ablation, the mechanism of these prolonged effects remains unclear. Proposed explanations include changes in the gut microbiome, mucosal permeability, bile acid composition, and neuromodulation of the myenteric plexus. Reported adverse effects included gastrointestinal symptoms (nausea, diarrhea, abdominal pain, and oropharyngeal pain), systemic symptoms (malaise, fatigue, myalgia, and rash), and metabolic symptoms (hypoglycemia and hyperglycemia). The relationship between duodenal mucosal resurfacing and GERD symptoms is currently unknown.

\section{Bariatric Surgery and Gastroesophageal Reflux Disease}

Three primary procedures comprise the majority of all bariatric surgeries performed in the United States. These include the Rouxen-Y gastric bypass (RYGB), laparoscopic sleeve gastrectomy $(\mathrm{SG})$, and adjustable gastric band (AGB), which are represented in Figure $2 .{ }^{53}$ In 2013, SG surpassed RYGB as the most commonly performed bariatric surgery in North America and Asia. ${ }^{54}$ The rising popularity of SG presents a predicament to clinicians managing both obesity and GERD, as the effect of SG on reflux is disputed.

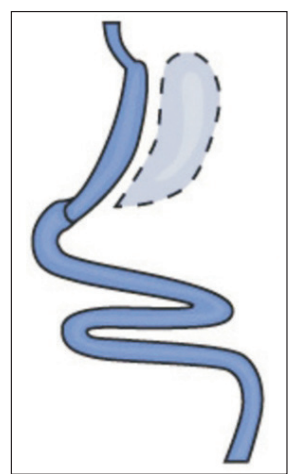

Sleeve gastrectomy

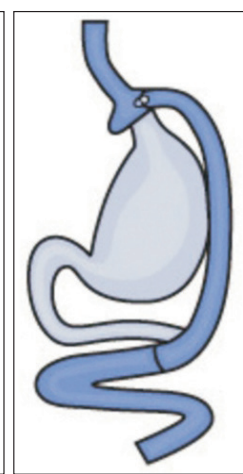

Roux-en-Y gastric bypass

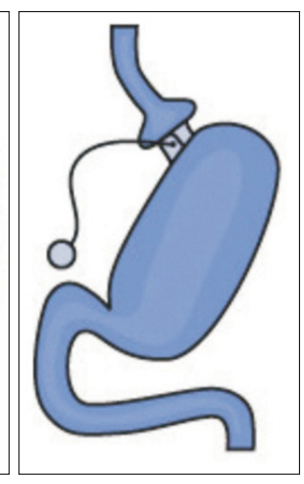

Adjustable gastric band
Figure 2. Common bariatric surgery options. Reproduced from the National Institute of Diabetes and Digestive and Kidney Diseases. ${ }^{53}$

\section{Laparoscopic Sleeve Gastrectomy}

Studies evaluating the relationship between SG and GERD have yielded conflicting results. A systematic review of 15 publications identified 4 studies which showed an increase and 7 studies which demonstrated a decrease in GERD symptoms after SG. ${ }^{55}$ A separate retrospective study of 4832 patients undergoing SG found that among 2150 (44.5\%) with pre-operative GERD, 84.1\% remained symptomatic after surgery. Among those without previous GERD, 8.6\% developed new symptoms. Furthermore, the presence of GERD pre-operatively was associated with decreased weight loss, increased post-operative complications, and the need for revisional surgery. ${ }^{56}$ A study of 109 patients undergoing SG found that at 18 months post-surgery, there was an increase in reflux prevalence from $33.0 \%$ to $44.0 \% .^{57}$ Recently, a prospective clinical trial comparing the incidence of GERD after SG and RYGB found that the incidence of GERD following SG was $66.6 \%$ and the mean DeMeester score increased from 10.9 to $40.2(P=$ 0.006). In comparison, the mean DeMeester score for RYGB did not significantly change $(P=0.700) .{ }^{58}$ Another study of 222 patients undergoing SG showed that $52.0 \%$ of patients complained of postoperative GERD symptoms, of which $73.0 \%$ were de novo. ${ }^{59}$ Also, of concern is an increase in post-operative asymptomatic reflux, as described by Pilone et al. ${ }^{60}$

Studies assessing long-term outcomes of SG have offered further evidence of worsening GERD symptoms. The Swiss Multicenter Bypass or Sleeve Study trial evaluated 5-year postoperative outcomes of SG versus RYGB ${ }^{61}$ They found that among patients with baseline GERD, symptom remission occurred in $25.0 \%$ of those undergoing SG compared to $60.4 \%$ of those undergoing RYGB (adjusted $P=0.002$ ). Additionally, $31.6 \%$ of patients without prior GERD developed de novo symptoms after SG compared to $10.7 \%$ after RYGB $(P=0.010)$. Another retrospective study of 130 patients undergoing SG reported that $8.5 \%$ required revision to RYGB for symptomatic reflux. ${ }^{62}$ In addition to reflux, a study of $110 \mathrm{SG}$ patients reported significant post-operative increases in erosive esophagitis, PPI use, and Barrett's esophagus. ${ }^{63}$

The relationship between SG and GERD is concerning given that obese patients have higher rates of acid reflux and its complications. The prevalence of GERD among patients considered for bariatric surgery is thought to be as high as $73.0 \% .^{64}$ Additionally, obese patients are at increased risk of erosive esophagitis, with an adjusted risk ratio of 1.76 for persons with a BMI $>25 \mathrm{~kg} / \mathrm{m}^{2}$. SG is thought to increase GERD symptoms by 2 key mechanisms. First, division of the crural ligaments and disruption of the angle 
of His results in decrease in LES basal pressure. Second, the ensuing gastric pouch has low compliance, which increases intragastric pressure. Together, the 2 mechanisms favor the reflux of gastric acid into the esophageal lumen. ${ }^{66}$

In an effort to make SG more accessible to GERD patients, some experts have advocated for concurrent hiatal hernia exploration and repair. A study evaluating the role of hiatal hernia repair during SG reported that $22.9 \%$ of participants without hiatal hernia repair developed de novo GERD, as compared to $0.0 \%$ of patients who underwent concurrent hiatal hernia repair. ${ }^{67}$ Another study examined 134 patients undergoing SG. Of these patients, 66 (49.2\%) had a pre-operative diagnosis of GERD, and 34 (25.3\%) had a hiatal hernia detected during surgery. Following SG with hiatal hernia repair, only 2 patients $(1.5 \%)$ had GERD symptoms at 6-12 months follow-up. ${ }^{68}$ In contrast, Santonicola et $\mathrm{al}^{69}$ demonstrated that hiatal hernia repair in patients undergoing $\mathrm{SG}$ was associated with more GERD than in those undergoing SG without hiatal hernia repair. In their study, 78 patients undergoing SG with hiatal hernia repair were compared to 102 patients undergoing SG alone. The prevalence of GERD pre-operatively was the same between both groups. Following surgery, patients who underwent concurrent $\mathrm{SG}$ and hiatal hernia repair reported more frequent and intense GERD symptoms compared to those who underwent SG alone.

A novel surgical technique for patients with pre-operative reflux is the N-sleeve gastrectomy, which consists of an SG with Nissen fundoplication. While trial data are lacking, preliminary cohort studies reveal promising results. A study of 70 patients with pre-operative GERD found that after N-sleeve gastrectomy, the prevalence of erosive esophagitis decreased from $99.0 \%$ to $30.0 \%$ and symptomatic reflux decreased from $76.0 \%$ to $1.0 \% .^{70}$ Another emerging technique for managing reflux following SG is magnetic sphincter augmentation. While not restricted to obese patients, a small retrospective review at a single academic medical center demonstrated a good response. ${ }^{71}$

The role of upper endoscopy prior to SG remains controversial with studies yielding conflicting results. A retrospective review of 1555 patients undergoing SG demonstrated that in asymptomatic patients, notable findings on pre-operative endoscopy were rare. ${ }^{72}$ Among symptomatic patients, the majority had hiatal hernias and their GERD symptoms resolved following SG with a crural repair. In contrast, a prospective study of 142 patients planned for bariatric surgery found that $47.1 \%$ of participants had significant lesions identified on upper endoscopy irrespective of symptoms. ${ }^{73}$ The authors concluded that without pre-operative endoscopy, $87.0 \%$ of erosive esophagitis cases would be missed.

\section{Roux-en-Y Gastric Bypass}

Although SG has overtaken RYGB as the most common bariatric procedure in North America, RYGB remains popular worldwide. Overall, $45.0 \%$ of all bariatric procedures performed globally in 2013 were RYGB. ${ }^{54}$ RYGB has been shown to be equivalent to SG in terms of weight loss, however, it outperforms SG in regards to improvement of co-morbidities. ${ }^{74,75}$ RYGB has consistently demonstrated a benefit in GERD and remains the surgery of choice in symptomatic patients. A prospective study of 239 GERD patients undergoing RYGB reported that $94.0 \%$ of the participants had improved symptoms at 9 months follow-up. ${ }^{76}$ Furthermore, medication usage decreased from $30.0 \%$ pre-operatively to $5.0 \%$ postoperatively and the improvement in GERD symptoms was shown to be independent of weight loss. Subsequent studies have corroborated the benefit of RYGB, with most experts recommending RYGB as the first line bariatric surgery for patients with GERD. ${ }^{77}$ Given the improvement in GERD symptoms, it can also be inferred that RYGB improves Barrett's esophagus. A systematic review of 8 studies encompassing 10779 patients undergoing RYGB revealed significant regression of both short and long-segment Barrett's esophagus at $>1$-year follow-up. ${ }^{78}$

The improvement in acid reflux following RYGB can be attributed to several mechanisms. First, the decrease in gastric pouch size results in less acid production and a smaller reservoir for regurgitation. ${ }^{79}$ Second, significant weight loss following surgery results in decreased intragastric pressure. ${ }^{77}$ Finally, the absence of direct communication between the duodenum and esophagus reduces duodenal gastric reflux. ${ }^{80}$ However, if a short Roux limb is present, the patient may be prone to esophageal ulceration secondary to alkaline reflux. $^{77,80}$

A potential alternative to RYGB is the one anastomosis gastric bypass (OAGB). A retrospective cohort study of 55 patients requiring revisional bariatric surgery after failed SG evaluated shortterm outcomes of RYGB versus OAGB.$^{81}$ Of the participants, $33.0 \%$ required revisional surgery for intractable reflux symptoms. While the reflux symptom score did not change significantly for the OAGB group, the overall trend was towards improvement and the study was notably underpowered. Further research is required to determine whether OAGB is a feasible alternative to RYGB for patients requiring revisional surgery following SG. For patients with GERD failing to improve after RYGB, a rescue modified Nissen fundoplication can be considered. While data is limited, a case series of 6 patients with persistent symptoms following RYGB demonstrated resolution of symptoms 1 month following surgery. ${ }^{82}$ 


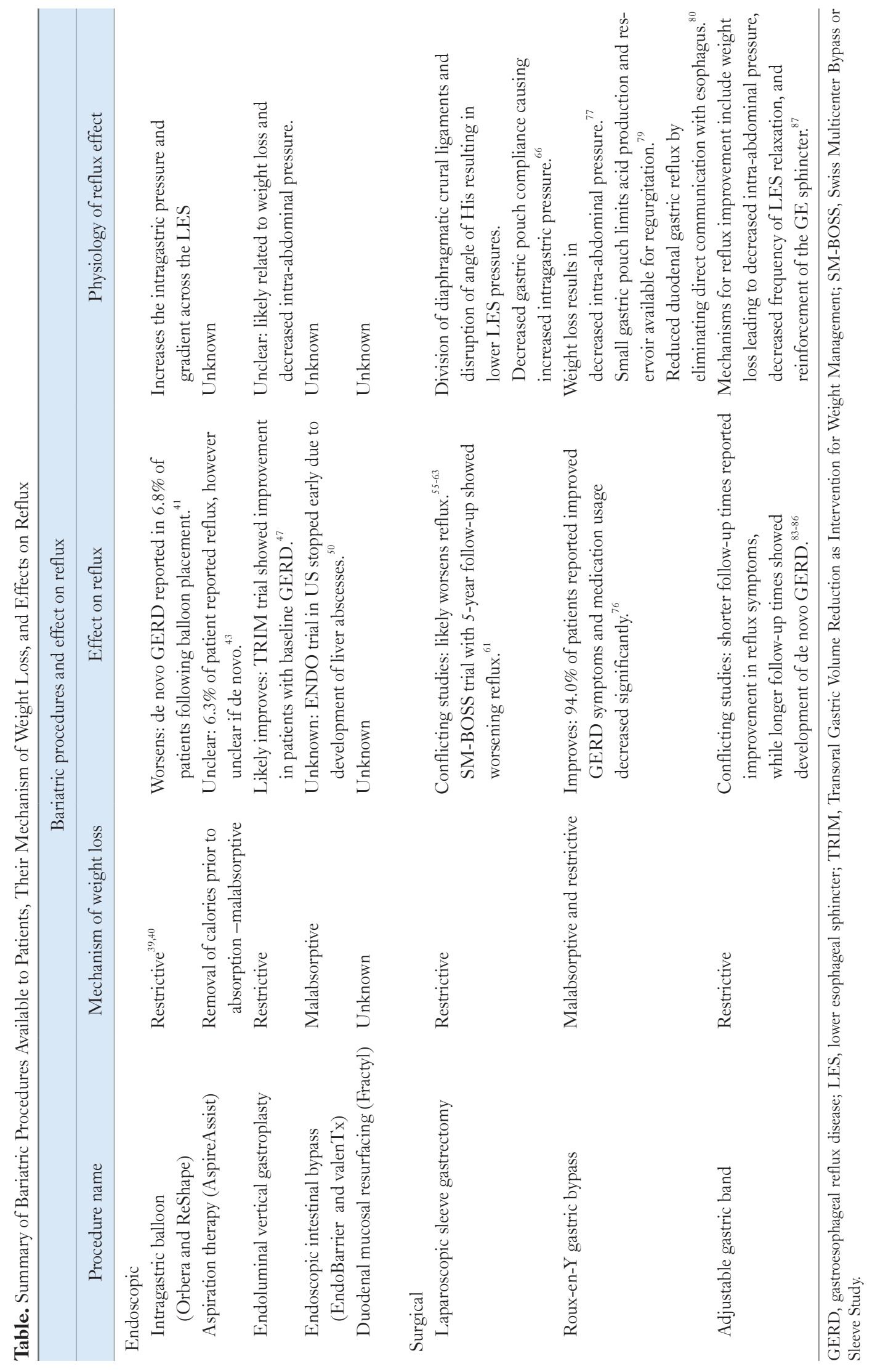




\section{Adjustable Gastric Bands}

The popularity of AGB has decreased from $68.0 \%$ of global weight loss surgeries in 2008 to $10.0 \%$ in $2013 .{ }^{83}$ Similar to SG, the effect of AGB on GERD remains uncertain. A study by Dixon and Obrien $^{83}$ evaluated the prevalence of GERD after AGB surgery in 48 patients. At 12-months follow-up, $76.0 \%$ participants reported complete resolution of their GERD symptoms, $14.0 \%$ had improved symptoms, and only 2 patients (4\%) described increased reflux symptoms. ${ }^{83}$ Similar results were published by Lew et al. ${ }^{84}$ The authors studied 77 patients with GERD, who underwent esophageal manometry before AGB. At 6- and 12-months followup, $100.0 \%$ of the patients with abnormal manometry had complete resolution of their GERD symptoms as compared to $92.0 \%$ of patients with normal manometry. ${ }^{84}$ In contrast, studies with follow-up periods beyond 1-year reported less satisfactory results. Korenkov et $\mathrm{al}^{85}$ evaluated GERD symptoms in 21 patients following AGB. At 22 months follow-up, among 6 patients with preoperative GERD, 3 had resolution of their symptoms and 3 reported no change. An additional 3 patients without preoperative GERD developed new symptoms. ${ }^{85}$ These findings were supported by a systematic review of 20 studies encompassing 3307 patients who underwent AGB. ${ }^{86}$ While the proportion of patients with GERD symptoms decreased from $32.9 \%$ to $7.7 \%$ following surgery, $15.0 \%$ of patients developed de novo reflux. Likewise, the prevalence of erosive esophagitis decreased from $33.3 \%$ to $27.0 \%$ postoperatively, however new erosive esophagitis developed in $22.9 \%$ of patients. ${ }^{86}$

The effect of AGB on reflux requires further study. A study by Woodman et $\mathrm{al}^{87}$ offers several potential mechanisms by which AGB may reduce reflux and improve GERD symptoms. These include weight loss leading to decreased intragastric pressure, reinforcement of the gastroesophageal sphincter, and decreased frequency of transient LES relaxation. ${ }^{87}$ Table summarizes the various bariatric procedures available to patients, their mechanism of weight loss, and effects on reflux.

\section{What Is Still Unknown?}

Further studies are needed to evaluate how obesity affects the response to medical and endoscopic reflux therapy. It is also unclear what pre-operative diagnostic workup obese patients with suspected GERD should undergo. Presently, the society guidelines are inconsistent and any testing that is undertaken consists solely of an upper endoscopy, which may be insufficient to predict post-operative symptoms. Moreover, the long-term impact of bariatric surgery on
GERD symptoms and esophageal motility remains to be fully elucidated.

\section{Conclusions}

Obesity and its associated comorbid conditions remain a burden on healthcare systems worldwide. As the prevalence of obesity increases, the same can be expected of GERD. Thus, it is important to understand the relationship between obesity and GERD to appropriately manage both conditions. If left untreated, GERD can lead to erosive esophagitis, peptic strictures, Barrett's Esophagus, and ultimately esophageal adenocarcinoma. ${ }^{88}$

Novel endoluminal weight loss procedures and bariatric surgeries provide tools for managing obesity that are superior to noninvasive modalities. ${ }^{89}$ Comorbid conditions often improve and sometimes resolve in patients who achieve significant weight loss. ${ }^{90}$ Yet, the effect of weight loss surgeries and endoluminal procedures on GERD symptoms remains uncertain, particularly regarding SG. While a few studies have shown that SG with hiatal hernia repair may avoid the development of postoperative GERD, most experts advocate for avoiding SG in patients with severe erosive esophagitis or Barrett's esophagus. It remains difficult to ascertain which patients will have worsening of reflux following SG and which will improve. Currently, RYGB remains the surgery of choice for obese patients with GERD. As new modalities for weight loss become available, additional studies are needed to determine their effect on GERD and reflux symptoms. This remains especially true for endoluminal bariatric procedures, for which little data currently exists.

\section{Financial support: None.}

\section{Conflicts of interest: None.}

Author contributions: Ofer Z Fass performed the literature review, composed the manuscript, and assisted with revisions; and Hiroshi Mashimo assisted with the literature review, revised the manuscript, and provided expert guidance.

\section{References}

1. Cawley J, Meyerhoefer C. The medical care costs of obesity: an instrumental variables approach. J Health Econ 2012;31:219-230.

2. Ogden CL, Carroll MD, Kit BK, Flegal KM. Prevalence of childhood and adult obesity in the United States, 2011-2012. JAMA 2014;311:806814.

3. Levi J, Segal LM, St Laurent R, Lang A, Rayburn J. F as in fat: how obesity threatens America's future 2012. Washington: Trust for America's 
Health/Robert Wood Johnson Foundation 2012.

4. Wang YC, McPherson K, Marsh T, Gortmaker SL, Brown M. Health and economic burden of the projected obesity trends in the USA and the UK. Lancet 2011;378:815-825.

5. Delshad SD, Almario CV, Chey WD, Spiegel BMR. Prevalence of Gastroesophageal Reflux Disease and Proton Pump Inhibitor-Refractory Symptoms. Gastroenterology 2020;158:1250-1261, e2.

6. Vakil N, van Zanten SV, Kahrilas P, Dent J, Jones R, Global Consensus Group. The Montreal definition and classification of gastroesophageal reflux disease: a global evidence-based consensus. Am J Gastroenterol 2006;101:1900-1920.

7. Kahrilas PJ, Hughes N, Howden CW. Response of unexplained chest pain to proton pump inhibitor treatment in patients with and without objective evidence of gastro-oesophageal reflux disease. Gut 2011;60:14731478.

8. Kahrilas PJ. Complications of gastroesophageal reflux in adults. Volume 2020. Waltham, MA: UpToDate 2020.

9. Shaheen N, Ransohoff DF. Gastroesophageal reflux, Barrett esophagus, and esophageal cancer: scientific review. JAMA 2002;287:1972-1981.

10. Jacobson BC, Somers SC, Fuchs CS, Kelly CP, Camargo Jr CA. Bodymass index and symptoms of gastroesophageal reflux in women. $\mathrm{N}$ Engl J Med 2006;354:2340-2348.

11. Singh M, Lee J, Gupta N, et al. Weight loss can lead to resolution of gastroesophageal reflux disease symptoms: a prospective intervention trial. Obesity (Silver Spring) 2013;21:284-290.

12. Thrift AP, Shaheen NJ, Gammon MD, et al. Obesity and risk of esophageal adenocarcinoma and Barrett's esophagus: a Mendelian randomization study. JNCI: J Natl Cancer Inst 2014;106:dju252.

13. Wilson LJ, Ma W, Hirschowitz BI. Association of obesity with hiatal hernia and esophagitis. Am J Gastroenterol 1999;94:2840-2844.

14. Kahrilas PJ, Lin S, Chen J, Manka M. The effect of hiatus hernia on gastro-oesophageal junction pressure. Gut 1999;44:476-482.

15. Fein M, Ritter MP, DeMeester TR, et al. Role of the lower esophageal sphincter and hiatal hernia in the pathogenesis of gastroesophageal reflux disease. J Gastrointest Surg 1999;3:405-410.

16. Polomsky M, Peters J, Schwartz S. Hiatal hernia and disorders of the spine: a historical perspective. Dis Esophagus 2012;25:367-372.

17. Che F, Nguyen B, Cohen A, Nguyen NT. Prevalence of hiatal hernia in the morbidly obese. Surg Obes Relat Dis 2013;9:920-924.

18. Mercer CD, Rue C, Hanelin L, Hill L. Effect of obesity on esophageal transit. Am J Surg 1985;149:177-181.

19. Pandolfino JE, El-Serag HB, Zhang Q, Shah N, Ghosh SK, Kahrilas PJ. Obesity: a challenge to esophagogastric junction integrity. Gastroenterology 2006;130:639-649.

20. de Mello Del Grande L, Herbella FAM, Katayama RC, Lima WG, Patti MG. Transdiaphragmatic pressure gradient (TPG) has a central role in the pathophysiology of gastroesophageal reflux disease (GERD) in the obese and it correlates with abdominal circumference but not with body mass index (BMI). Obes Surg 2020;30:1424-1428.

21. Matsuzaki J, Suzuki H, Kobayakawa M, et al. Association of visceral fat area, smoking, and alcohol consumption with reflux esophagitis and Barrett's esophagus in Japan. PLoS One 2015;10:e0133865.
22. Valipour A, Makker HK, Hardy R, Emegbo S, Toma T, Spiro SG. Symptomatic gastroesophageal reflux in subjects with a breathing sleep disorder. Chest 2002;121:1748-1753.

23. Lim KG, Morgenthaler TI, Katzka DA. Sleep and nocturnal gastroesophageal reflux: an update. Chest 2018;154:963-971.

24. Ing AJ, Ngu MC, Breslin AB. Obstructive sleep apnea and gastroesophageal reflux. The Ame J Med 2000;108(suppl 1):120-125.

25. Tawk M, Goodrich S, Kinasewitz G, Orr W. The effect of 1 week of continuous positive airway pressure treatment in obstructive sleep apnea patients with concomitant gastroesophageal reflux. Chest 2006;130:10031008.

26. Shepherd K, Hillman D, Holloway R, Eastwood P. Mechanisms of nocturnal gastroesophageal reflux events in obstructive sleep apnea. Sleep Breath 2011;15:561-570.

27. Schey R, Dickman R, Parthasarathy S, et al. Sleep deprivation is hyperalgesic in patients with gastroesophageal reflux disease. Gastroenterology 2007;133:1787-1795.

28. Nebel OT, Castell DO. Inhibition of the lower oesophageal sphincter by fat - a mechanism for fatty food intolerance. Gut 1973;14:270-274.

29. Fox M, Barr C, Nolan S, Lomer M, Anggiansah A, Wong T. The effects of dietary fat and calorie density on esophageal acid exposure and reflux symptoms. Clin Gastroenterol Hepatol 2007;5:439-444, e431.

30. Lacy BE, Weiser K, Chertoff J, et al. The diagnosis of gastroesophageal reflux disease. Am J Med 2010;123:583-592.

31. Vakil NB, Traxler B, Levine D. Dysphagia in patients with erosive esophagitis: prevalence, severity, and response to proton pump inhibitor treatment. Clin Gastroenterol Hepatol 2004;2:665-668.

32. Madanick RD. Extraesophageal presentations of GERD: where is the science? Gastroenterology Clinics 2014;43:105-120.

33. Abdelkader NA, Montasser IF, Bioumy EE, Saad WE. Impact of anthropometric measures and serum leptin on severity of gastroesophageal reflux disease. Dis Esophagus 2015;28:691-698.

34. Rubenstein JH, Morgenstern H, McConell D, et al. Associations of diabetes mellitus, insulin, leptin, and ghrelin with gastroesophageal reflux and Barrett's esophagus. Gastroenterology 2013;145:1237-1244, e1-e5.

35. Iwasaki E, Suzuki H, Sugino Y, et al. Decreased levels of adiponectin in obese patients with gastroesophageal reflux evaluated by videoesophagography: possible relationship between gastroesophageal reflux and metabolic syndrome. J Gastroenterol Hepatol 2008;23(suppl 2):S216-S221.

36. Buchwald H, Oien DM. Metabolic/bariatric surgery worldwide 2011. Obes Surg 2013;23:427-436.

37. Ryou M, Ryan MB, Thompson CC. Current status of endoluminal bariatric procedures for primary and revision indications. Gastrointest Endosc Clin N Am 2011;21:315-333.

38. Nieben OG, Harboe H. Intragastric balloon as an artificial bezoar for treatment of obesity. Lancet 1982;319:198-199.

39. Lim LRB, Blackburn GL, Jones DB. Benchmarking best practices in weight loss surgery. Current problems in surgery 2010;47:79.

40. Vargas EJ, Bazerbachi F, Calderon G, et al. Changes in time of gastric emptying after surgical and endoscopic bariatrics and weight loss: a systematic review and meta-analysis. Clin Gastroenterol Hepatol 2020;18:57-68, e55. 
41. Al-Momen A, El-Mogy I. Intragastric balloon for obesity: a retrospective evaluation of tolerance and efficacy. Obes Surg 2005;15:101-105.

42. AspireAssist. FDA website - newly approved devices. Available from URL: https://www.fda.gov/news-events/press-announcements/fdaapproves-aspireassist-obesity-device (accessed 26 Nov 2020).

43. Summary of safety and effectiveness data for the aspire assist. Available from URL: https://www.accessdata.fda.gov/cdrh_docs/pdf15/p150024b. $\operatorname{pdf}($ accessed 26 Nov 2020).

44. Majumder S, Birk J. A review of the current status of endoluminal therapy as a primary approach to obesity management. Surg Endosc 2013;27:2305-2311.

45. Hedjoudje A, Abu Dayyeh BK, Cheskin LJ, et al. Efficacy and safety of endoscopic sleeve gastroplasty: a systematic review and meta-analysis. Clin Gastroenterol Hepatol 2020;18:1043-1053, e4.

46. Fogel R, De Fogel J, Bonilla Y, De La Fuente R. Clinical experience of transoral suturing for an endoluminal vertical gastroplasty: 1-year followup in 64 patients. Gastrointest Endosc 2008;68:51-58.

47. Brethauer SA, Chand B, Schauer PR, Thompson CC. Transoral gastric volume reduction as intervention for weight management: 12-month follow-up of TRIM trial. Surg Obes Relat Dis 2012;8:296-303.

48. Fayad L, Adam A, Schweitzer M, et al. Endoscopic sleeve gastroplasty versus laparoscopic sleeve gastrectomy: a case-matched study. Gastrointest Endosc 2019;89:782-788.

49. Koehestanie P, de Jonge C, Berends FJ, Janssen IM, Bouvy ND, Greve JW. The effect of the endoscopic duodenal-jejunal bypass liner on obesity and type 2 diabetes mellitus, a multicenter randomized controlled trial. Ann Surg 2014;260:984-992.

50. Ruban A, Ashrafian H, Teare JP. The EndoBarrier: duodenal-jejunal bypass liner for diabetes and weight loss. Gastroenterol Res Pract 2018;2018:7823182.

51. Cherrington AD, Rajagopalan H, Maggs D, Devière J. Hydrothermal duodenal mucosal resurfacing: role in the treatment of metabolic disease. Gastrointest Endosc Clin N Am 2017;27:299-311.

52. van Baar ACG, Holleman F, Crenier L, et al. Endoscopic duodenal mucosal resurfacing for the treatment of type 2 diabetes mellitus: one year results from the first international, open-label, prospective, multicentre study. Gut 2020;69:295-303.

53. National Institute of Diabetes and Digestive and Kidney Diseases. Types of bariatric surgery. Available from URL: https://www.niddk.nih.gov/ health-information/weight-management/bariatric-surgery/types\# gastricbypass (accessed 26 Nov 2020).

54. Angrisani L, Santonicola A, Iovino P, Formisano G, Buchwald H, Scopinaro N. Bariatric surgery worldwide 2013. Obes Surg 2015;25:18221832.

55. Chiu S, Birch DW, Shi X, Sharma AM, Karmali S. Effect of sleeve gastrectomy on gastroesophageal reflux disease: a systematic review. Surg Obes Relat Dis 2011;7:510-515.

56. DuPree CE, Blair K, Steele SR, Martin MJ. Laparoscopic sleeve gastrectomy in patients with preexisting gastroesophageal reflux disease : a national analysis. JAMA Surg 2014;149:328-334.

57. Viscido G, Gorodner V, Signorini F, Navarro L, Obeide L, Moser F. Laparoscopic sleeve gastrectomy: endoscopic findings and gastroesopha- geal reflux symptoms at 18-month follow-up. J Laparoendosc Adv Surg Tech A 2018;28:71-77.

58. Raj PP, Bhattacharya S, Misra S, et al. Gastroesophageal refluxrelated physiologic changes after sleeve gastrectomy and Roux-en-Y gastric bypass: a prospective comparative study. Surg Obes Relat Dis 2019;15:1261-1269.

59. Borbély Y, Schaffner E, Zimmermann L, et al. De novo gastroesophageal reflux disease after sleeve gastrectomy: role of preoperative silent reflux. Surg Endosc 2019;33:789-793.

60. Pilone V, Tramontano S, Renzulli M, Zulli C, Schiavo L. Gastroesophageal reflux after sleeve gastrectomy: new onset and effect on symptoms on a prospective evaluation. Obes Surg 2019;29:3638-3645.

61. Peterli R, Wölnerhanssen BK, Peters T, et al. Effect of laparoscopic sleeve gastrectomy vs laparoscopic Roux-en-Y gastric bypass on weight loss in patients with morbid obesity: the SM-BOSS randomized clinical trial. JAMA 2018;319:255-265.

62. Madhok BM, Carr WR, McCormack C, et al. Preoperative endoscopy may reduce the need for revisional surgery for gastro-oesophageal reflux disease following laparoscopic sleeve gastrectomy. Clin Obes 2016;6:268272.

63. Sebastianelli L, Benois M, Vanbiervliet G, et al. Systematic endoscopy 5 years after sleeve gastrectomy results in a high rate of Barrett's esophagus: results of a multicenter study. Obes Surg 2019;29:1462-1469.

64. Merrouche M, Sabaté JM, Jouet P, et al. Gastro-esophageal reflux and esophageal motility disorders in morbidly obese patients before and after bariatric surgery. Obes Surg 2007;17:894-900.

65. Hampel H, Abraham NS, El-Serag HB. Meta-analysis: obesity and the risk for gastroesophageal reflux disease and its complications. Ann of Intern Med 2005;143:199-211.

66. El-Hadi M, Birch DW, Gill RS, Karmali S. The effect of bariatric surgery on gastroesophageal reflux disease. Can J Surg 2014;57:139-144.

67. Soricelli E, Iossa A, Casella G, Abbatini F, Calì B, Basso N. Sleeve gastrectomy and crural repair in obese patients with gastroesophageal reflux disease and/or hiatal hernia. Surg Obes Relat Dis 2013;9:356-361.

68. Daes J, Jimenez ME, Said N, Daza JC, Dennis R. Laparoscopic sleeve gastrectomy: symptoms of gastroesophageal reflux can be reduced by changes in surgical technique. Obes Surg 2012;22:1874-1879.

69. Santonicola A, Angrisani L, Cutolo P, Formisano G, Iovino P. The effect of laparoscopic sleeve gastrectomy with or without hiatal hernia repair on gastroesophageal reflux disease in obese patients. Surg Obes Relat Dis 2014;10:250-255.

70. Amor IB, Casanova V, Vanbiervliet G, et al. The Nissen-Sleeve (NSleeve): results of a cohort study. Obes Surg 2020;30:3267-3272.

71. Broderick RC, Smith CD, Cheverie JN, et al. Magnetic sphincter augmentation: a viable rescue therapy for symptomatic reflux following bariatric surgery. Surg Endosc 2020;34:3211-3215.

72. Salama A, Saafan T, El Ansari W, Karam M, Bashah M. Is routine preoperative esophagogastroduodenoscopy screening necessary prior to laparoscopic sleeve gastrectomy? review of 1555 cases and comparison with current literature. Obes Surg 2018;28:52-60.

73. Carabotti M, Avallone M, Cereatti F, et al. Usefulness of upper gastrointestinal symptoms as a driver to prescribe gastroscopy in obese 
patients candidate to bariatric surgery. A prospective study. Obes Surg 2016;26:1075-1080

74. Arapis K, Macrina N, Kadouch D, Ribeiro Parenti L, Marmuse JP, Hansel B. Outcomes of Roux-en-Y gastric bypass versus sleeve gastrectomy in super-super-obese patients (BMI $\geq 60 \mathrm{~kg} / \mathrm{m}^{2}$ ): 6-year follow-up at a single university. Surg Obes Relat Dis 2019;15:23-33.

75. Zhao H, Jiao L. Comparative analysis for the effect of Roux-en-Y gastric bypass vs sleeve gastrectomy in patients with morbid obesity: evidence from 11 randomized clinical trials (meta-analysis). Int J Surg 2019;72:216-223.

76. Nelson LG, Gonzalez R, Haines K, Gallagher SF, Murr MM. Amelioration of gastroesophageal reflux symptoms following Roux-en-Y gastric bypass for clinically significant obesity. Am Surg 2005;71:950-954.

77. Ikramuddin S. Surgical management of gastroesophageal reflux disease in obesity. Dig Dis Sci 2008;53:2318-2329.

78. Adil MT, Al-Taan O, Rashid F, et al. A systematic review and metaanalysis of the effect of Roux-en-Y gastric bypass on Barrett's esophagus. Obes Surg 2019;29:3712-3721.

79. Smith CD, Herkes SB, Behrns KE, Fairbanks VF, Kelly KA, Sarr MG. Gastric acid secretion and vitamin B12 absorption after vertical Roux-enY gastric bypass for morbid obesity. Ann Surg 1993;218:91-96.

80. Johnson WH, Fernanadez AZ, Farrell TM, et al. Surgical revision of loop ("mini”) gastric bypass procedure: multicenter review of complications and conversions to Roux-en-Y gastric bypass. Surg Obes Relat Dis 2007;3:37-41.

81. Chiappetta S, Stier C, Scheffel O, Squillante S, Weiner RA. Mini/one anastomosis gastric bypass versus Roux-en-Y gastric bypass as a second step procedure after sleeve gastrectomy-a retrospective cohort study. Obes Surg 2019;29:819-827.

82. Colpaert J, Horevoets J, Maes L, Uijtterhaegen G, Dillemans B. Surgical treatment of therapy-resistant reflux after Roux-en-Y gastric bypass. A case series of the modified Nissen fundoplication. Acta Chir Belg 2019;120:291-296.

83. Dixon JB, O'Brien PE. Gastroesophageal reflux in obesity: the effect of lap-band placement. Obes Surg 1999;9:527-531.

84. Lew JI, Daud A, DiGorgi MF, Olivero-Rivera L, Davis DG, Bessler M. Preoperative esophageal manometry and outcome of laparoscopic adjustable silicone gastric banding. Surg Endosc 2006;20:1242-1247.

85. Korenkov M, Köhler L, Yücel N, et al. Esophageal motility and reflux symptoms before and after bariatric surgery. Obes Surg 2002;12:72-76.

86. de Jong JR, Besselink MGH, van Ramshorst B, Gooszen HG, Smout AJPM. Effects of adjustable gastric banding on gastroesophageal reflux and esophageal motility: a systematic review. Obes Rev 2010;11:297-305.

87. Woodman G, Cywes R, Billy H, et al. Effect of adjustable gastric banding on changes in gastroesophageal reflux disease (GERD) and quality of life. Curr Med Res Opin 2012;28:581-589.

88. Zagari RM, Fuccio L, Wallander MA, et al. Gastro-oesophageal reflux symptoms, oesophagitis and Barrett's oesophagus in the general population: the Loiano-Monghidoro study. Gut 2008;57:1354-1359.

89. Schauer PR, Kashyap SR, Wolski K, et al. Bariatric surgery versus intensive medical therapy in obese patients with diabetes. N Engl J Med 2012;366:1567-1576.

90. Maggard MA, Shugarman LR, Suttorp M, et al. Meta-analysis: surgical treatment of obesity. Ann Intern Med 2005;142:547-559. 